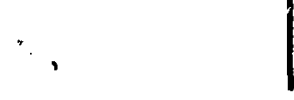

LA-UR $-74-56$
-

Cont $-740104-18$

TITLE: FIRST WALL FLUXES IN A THETA PINCH FEASIBILITY/D-T EAPERIMENT

AUTHOR(S): K. I. Thomassen and T. A. Oliphant

SUBMITTED TO: Conference on Surface Effects in Controlled Thermonuclear Devices and Reactors, Argonne National Laboratory, January 10-12, 1974

8y acceptance of this article for publication, the publisher recognizes the Government's (license) rights in any copyright and the Government and its authorized representatives have unrestricted right to reproduce in vahole or in part said article under ony copyright secured by the publisher.

The Los Alsinos Scientific Laboratory requests that the publisher identify this article as work performed under the auspices of the U.S. Atomic Energy Cormmission. sponsored by the United Siates Govern Atomic Enerby the United States nor the United States Atomic Ener any of Commission, nor any of their employees, nor any of their contractors, subcontractors, of thetr ensumes any makes any warranty, exprass or for the accuracy, comlegal liability or responsibuly information, apparutus, pleteness or usefulness of any infepresents that its use

product or procese privately owned rights. 


\author{
K. I. Thomassen, T. A. Oliphant \\ University of California, Los Alamos Scientific Laboratory \\ Los Alamos, New Mexico
}

\title{
Introduction
}

The first wall of a D-T burning theta pinch is subjected to electrical and thermal stresses which combine to present materials constraints of a unique nature in fusion devices. In this paper we address those constraints for the ITR (Physics Test Reactor) which will be designed to first demonstrate scientific feasibility and later be converted to handle tritium for $D-T$ burning experiments. The wall is subjected to fluxes of neutrons, photons, and particles, the last of these being the most important in the PTR, hence an estimation of their magnitude is required for design purposes.

A quantitative assessment of the wall problem requires scaling information from wall stabilized Scrllac experiments to specify the size of the experiment, implosion heating physics to determine the necessary electric field stress on the insulator to achieve ignition after magnetic compression, and a thermonuclear burn calculation specifying the time history of neutron and bremsitrahlung production and plasma internal energy. A concise statement of the scaling laws and their basis in experiment were recently given by Freidberg and Ribe, (1) and further verification will be forthcoming in the soon to be completed toroidal scyllac experiment.

The physics of the implosion process has been explored with both theoretical $(2,3)$ and computer simulation (4) models, and will soon come under experimental scrutiny in the implosion heating experiment and the staging experiment. (5) The thermonuclear burn code of Oliphant, (6) used in the design of the reference theta pinch reactor (RTPR), gives all the required plasma and radiation data except the particle fluxes. The particle flux must be estimated from plasma incerial energy and the expansion of the plasma in the decaying magnetic field. Diffusion of the magnetic field into the plasma limits the rapid loss of plasma after the coltun expands to the wall and the flux between the wall and the plasma boundary disappears. The results of this calculation giving the wall flux will be described. 
As present1y envisaged, the Physics Test Reactor is a large aspect ratio torus, driven by separate implosion heating and compression coils. A schematic layout is shown in Fig. 1. The aspect ratio is determined by considerations of stability and equilibrium while the minor radius " $\mathrm{b}$ " is made as sma11 as possible yet consistent with the formation of a we11-defined piston of thickness $c / \omega_{p i} \ll b$. A sufficiently large value of $b$ is also necessary to present a reasonable inductance to the implosion circuitry. These criteria lead to a major radius $R=35 \mathrm{~m}$ and $a$ minor radius $b=0.1 \mathrm{~m}$.

The implosion heating coil is located inside the compression coil as shown in Fig. 2, and the first wall at $b=0.1 \mathrm{~m}$ is inside the implosion coil. The two field coils produce the magnetic field tine variation shown in Fig. 2. Electrical stress on the first wall is Induced by the large azimutahl electric field $(\sim 3 \mathrm{kV} / \mathrm{cm})$ needed to give the rapid initial magnetic field rise $(\sim 0.1 \mathrm{\mu sec})$.

Since the first wall must contain tritiun it must have sufficient mechanical integrity to ensure containment of this raaioactive gas. Fresent day quartz tubes, which are otherwise appropriate for the device, are therefore inadequate since they occasionally break when a crowbar fails.

A final requirement is that the tube endure the thermal stresses from particle and photon bombardment, and thar surface spallation and sputtering be minimized. The preliminary design of this tube calls for a segmented metal and insulator structure which allows radial penetration of magnetic flux. An exact configuration has not been selected, but it must meet the criteria described in this paper。

\section{Implosion Heating and Burning}

The PTR plaswa is brought to ignition by a combination of implosion heating and adiabatic compression. From the physics of implosion heating as described by a bounce model and free expansion after the contraction of the ions to their minimum radius, the plasma radius after the implosion process is determined. Further, when the initial filling pressure and azimuthal electric field are specified the temperature and magnetic field (Bs) values after the implosion process are uniquely determined. Specification of the magnetic field to which the plasma is compressed and of che initial electron temperature then yields the final electron and ion temperatures, the alpha particle production rate, the final plasma radius, the plasma internal energy, and the neutron and bremsstrahlung wal1 fluxes. These parameters are the output of a thermonuclear burn code developed by 01iphant (5) which follows the evolution of the ion, electron, and alpha particle distribution functions as the alphas are born and deposit their energy in the plasma. The plasma expands adiabatically against the magnetic field $(\beta=1)$, doing direct conversion work, and also cools by bremsstrahlung radiation. All these processes are treated self- 
PTR Burn Calculations for $1 \mathrm{msec}$ field risetime from $\mathrm{B}_{\mathrm{s}}$ to $60 \mathrm{kG}\left(\sim \mathrm{sin}^{2} \mathrm{~T}\right)$ and exponential decay $\left(\tau_{D}\right)$ thereafter.

$P_{0} \quad=$ filling pressure (mtorr)

$E_{\theta} \quad=$ shock field $\mathrm{kV} / \mathrm{cm}$

$\mathrm{f}=$ percent burnup (\%) $\begin{aligned} \mathrm{E}_{\text {INT }}= & \text { internal plasma energy density } \\ & \text { at first wall } \mathrm{J} / \mathrm{cm}^{2}\end{aligned}$
$E_{B R}=$ bremsstrahlung energy density at first wall $\mathrm{J} / \mathrm{cm}^{2}$

$\phi=$ neutron wall flux at first wall (peak) $10^{74} / \mathrm{cm}^{2}$ sec
$\underline{P}_{\text {o }}$

2

2

3

4

5

5

8

8

2

2

5

5

8

8

2

2

5

5

8

8 
consistently in following the plasma history.

The results of the application of this code to the PTR plasma are given in Table I for varlous filling pressures $\rho_{0}$ (m torr) and electric fields $E_{\theta}(\mathrm{kV} / \mathrm{cm})$. In each case the compression field reached $60 \mathrm{kG}$ and then decayed exponential with a time constant ${ }^{\top} \mathrm{d}$. The computed paraneters of interest here are fractional burnup, neutron and bremstrahlung wall fiux, and incernal plasma energy densicy. The latter is the peak energy achieved during burn, divided by the wall area surrounding the plasma. The rate at which the energy is deposited is separately determined from an MHD diffusion and heat transport calculation.

The first thing to note is that the major heat load at the first wall will come from the transport of the internal energy as the magnetic field decays. The bremsstrahlung load is not important, and the neutron flux will produce insignificant fluences during the life of the wall at the anticipated duty cycle.

To indicate the temporal behavior of the fluxes and energies in Table $I$, we have plotted in Figs. 3-5 the time variation of the internal energy $\left(\mathrm{J} / \mathrm{cm}^{2}\right)$, the bremsstrahlung flux, (watts $/ \mathrm{cm}^{2}$ ) and neutron $f 1 \mathrm{ux}\left(\mathrm{no}_{0} / \mathrm{cm}^{2} / \mathrm{sec}\right)$ for the case $\rho_{0}=5 \mathrm{~m}$ torr, $\mathrm{E}_{\theta}=3 \mathrm{kv} / \mathrm{cm}$. The internal energy is about $3.5 \mathrm{~J} / \mathrm{cm}^{2}$ when the plasma begins making substantial wall contact. Neutron currents exceed $10^{1.4} / \mathrm{cm}^{2} / \mathrm{sec}$ during the initial burn, and bremsstrahlung rates diminish from 13 watts $/ \mathrm{cm}^{2}$.

\section{Particle and Heat Transport}

To find the particle $f 1 u x$ and heat transport to the wall requires a separate calculation. We use a single fluid MHD model to follow the resistive diffusion of magnetic field into the plasma during the expansion, as described by the equation.

$$
\nabla \times(\eta \nabla \times H-\nabla \times B)=-\frac{\partial B}{\partial t}
$$

The resistivity $\eta$ is given by the classical spitzer value multiplied by an anonaly factor. The convective term is sma11 and neglected in the code. Pressure balance is maintained by demanding $\nabla \mathrm{p}=(\nabla \times \mathrm{H}) \times \mathrm{B}$.

Heat flow is included by modifying the usual adiabatic equation relating pressure and density as follows:

$$
\frac{\mathrm{dT}}{\mathrm{dt}}=(1-\gamma) \rho \mathrm{T} \frac{\mathrm{d}}{\mathrm{dt}}\left(\frac{1}{\rho}\right)+\frac{\gamma-1}{\mathrm{R} \rho} \nabla \cdot\left(u_{\perp} \nabla \mathrm{T}\right)
$$

The gas constant is $R$ and $x_{\perp}$ is the heat conductivity of ions as given by Spitzer.

$$
x_{\perp}=\frac{8\left(\pi m_{1} k\right)^{1 / 2} n^{2} e^{2} c^{2} \ln \Lambda}{3 B^{2} T^{1 / 2}} \operatorname{erg} / c m \sec { }^{\circ} K
$$


Along with the equation of state, $p=2 n \mathrm{kT}$, the solution of this set of equations gives the temperature, density, and magnetic field profiles. One can then calculate the particle and heat fluxes to the wall. The ions have an essentialiy Maxwellian velocity distribution while the alphas have a distribution from $3.5 \mathrm{MeV}$ down to nearly zero energy. The mean alpha energy can be obtained from the burn code, and for the $r$ uns in Table $I$ is typically $2 \mathrm{MeV}$.

In Fig. 6 the time variation of the particle flux and a normalized integrated wall flux is shown for the case $p_{0}=5$ mtorr, $E_{\theta}=3 \mathrm{kV} / \mathrm{cm}$. The initial conditions for the MHD code assumed a plastna radius corresponding to the minimun radius after compression, and a magnetic field of $60 \mathrm{kG}$ outside the plasma and $12 \mathrm{kG}$ inside the plasma. This sharp boundary plasma was allowed to expand as the field diffused and smeared the boundary. The $60 \mathrm{kG}$ field at the wall was diminished exponentially in $100 \mathrm{msec}$. An anomaly factor of 100 was put into the resistivity to smear the field profile but the heat conductivity value was the previously stated classical value. A more appropriate procedure would perhaps be to give che magnetic field boundary a finite transition width, since the ancmalous transport occurs mostiy in the implosion phase, and assume classical transport thereafter.

Note in Fig. 6 that the particle flux increases rapidly after $120 \mathrm{msec}$, we 11 after the bremsstrahlung flux has diminished, reaching $6 \times 10^{12} / \mathrm{cm}^{2} / \mathrm{sec}$ and lasting about 50 msec. As a rough approximation these partieles consist of ions and electrons at temperatures of about $2 \mathrm{KeV}$ and $0.38 \%$ alphas at an average energy of $2 \mathrm{MeV}$.

The heat flow from the ions is shown in Fig. 7, and this flow occurs over about 25 msec. The total conducted heat is 0.76 joules $/ \mathrm{cm}^{2}$, or $25 \%$ of the internal plasma energy when the plasma first strikes the wall. The remainder may be accounted for by the alpha particies which are 1000 times more energetic and 3.8 thousandths less numerous, hence account for 3.8 times the 0.76 joules $/ \mathrm{cm}^{2}$ or 2.89 joules $/ \mathrm{cm}^{2}$. This heat load will give about a $30^{\circ} \mathrm{K}$ temperature rise on an alumina first wall.

The results of this study are in substantial agreement with earlier analytical calculations by Burnett and Ellis (7) for a rather similar PTR design. One major discrepancy is in the particle wall flux, which we find is many orders of magnitude larger. However, they considered only the early time diffusion and hence estimated the minute quantities of ions, electrons, and alphas, which hit the wall during the burn. As seen here the majority of the particles cone later.

The studies here are quite preliminary but should provide adequate information for initial surface studies in a theta pinch PTR. More detailed analyses are being carried out. 
1. J. P. Freidberg and F. L. Ribe, "Scaling of Wal1-Stabilized Scyllac Experiments," Coments on Plasma Physics and Controlled Fusion, to be published.

2. J. P. Freidberg, R. L. Morse, and F. L。 Ribe, "Staged Theta Pinches With Implosion Heating," Texas Symposium on the Technology of Controlled Thermonuclear Fusion Experiments and the Engineering Aspects of Fusion Reactors, Nov。20-22, 1972.

3. T. A. Oliphant, "A Mixed Snowplow Bounce Mode1 for Shock Heating in a Staged Theta Pinch," Nuclear Fusion, submitted for publication.

4. C. Nielson, private communication.

5. F. I. Ribe, "Proposed Experiments on Heating, Staging, and Stabilization of Theta Pinches," Los Alamos Scientific Laboratory, LA-5026-P, Feb. 1973.

6. T. A. Oliphant, "Fuel Burnup and Direct Conversion of Energy in a D-T Plasma," in Proceedings of the B.N.E.S. Conference on Nuclear Fusion Reactors held at the UKAEA Culham Iaboratory 17-19 Sept. $196 y$ (UKAEA Culham Laboratory, 1969) p. 306.

7. S. C. Burnett and W. R. E11is, "Radiation and Wall Flux Calculations for a ThetaPinch Scy.entific Feasibility Experiment and a Prototype Reactor," Los Aiamos Scientific Laboratory, LA-4814-MS, Oct. 1971.

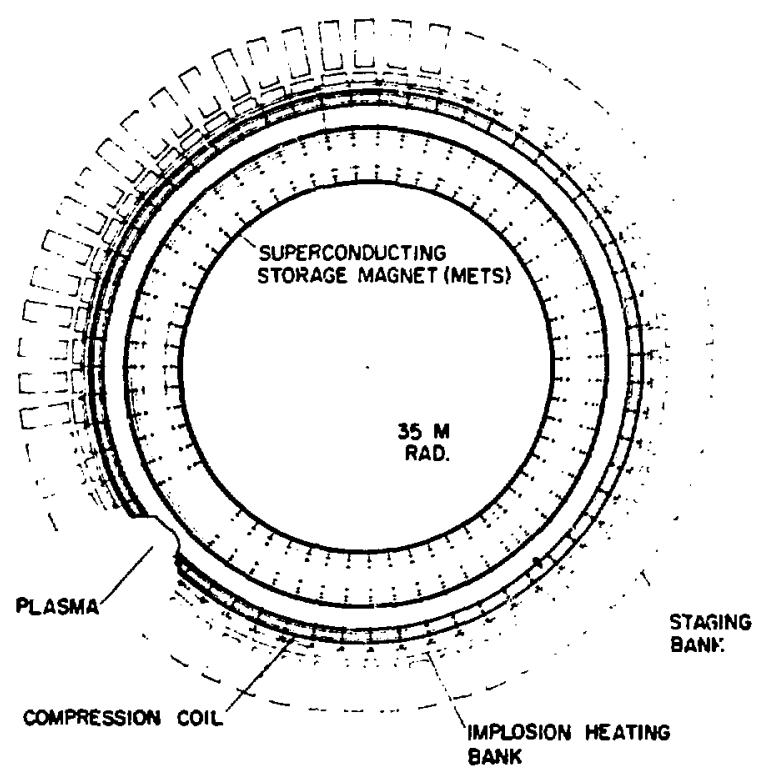

FIG. 1

Schematic layout of the Physics Test Reactor. 

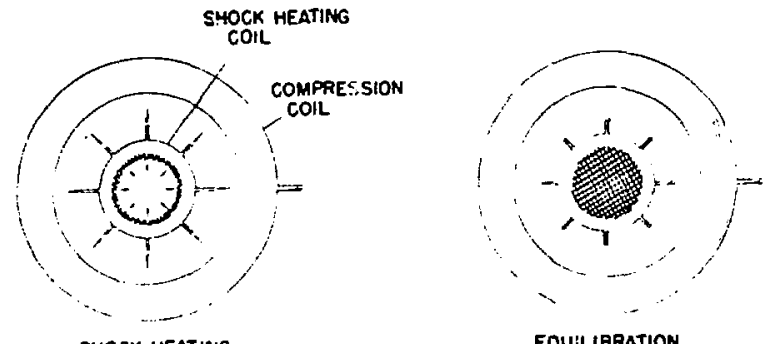

SHOCK HEATING

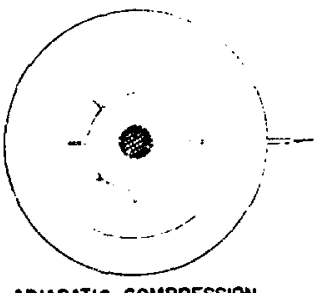

ADIAGATIC COMPRESSION
EQUILIBRATION

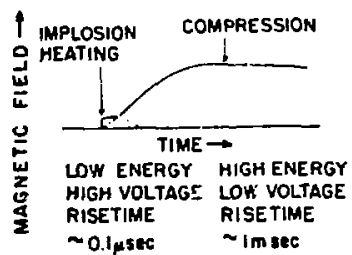

FIG. 2

Time variation of the magnetic field and the plasma processes leading to ignition.

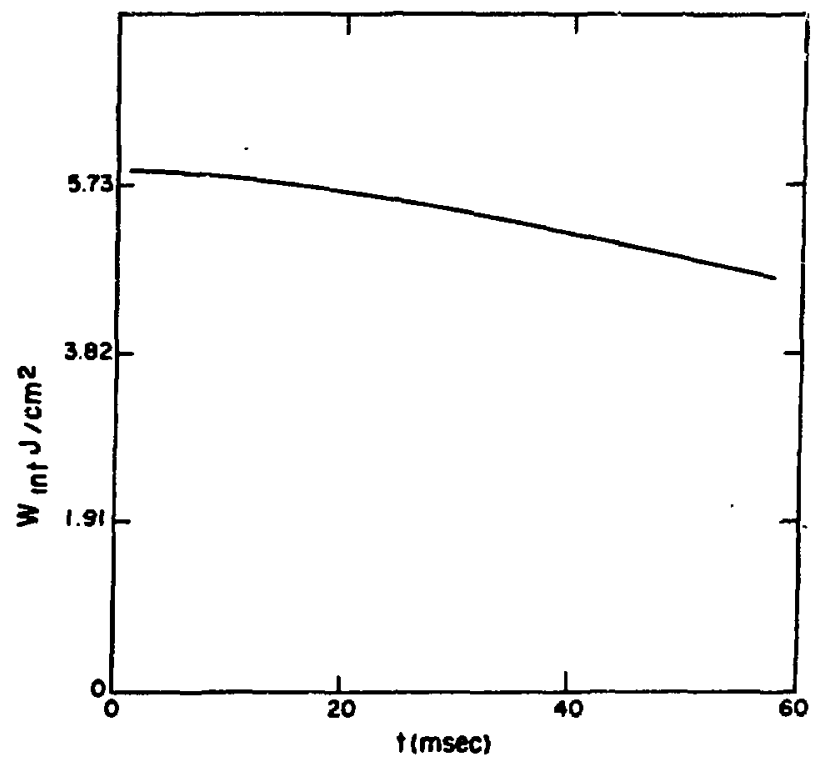

FIG. 3

Internal plasma energy after a 1 msec compression to ignition and $100 \mathrm{msec}$ decay of the $60 \mathrm{kG}$ external field for $\mathrm{P}_{0}=5$ mtorr and $E_{\theta}=3 \mathrm{kV} / \mathrm{cm}$. The energy is normalized to the wall area which will eventually receive this heat load. 


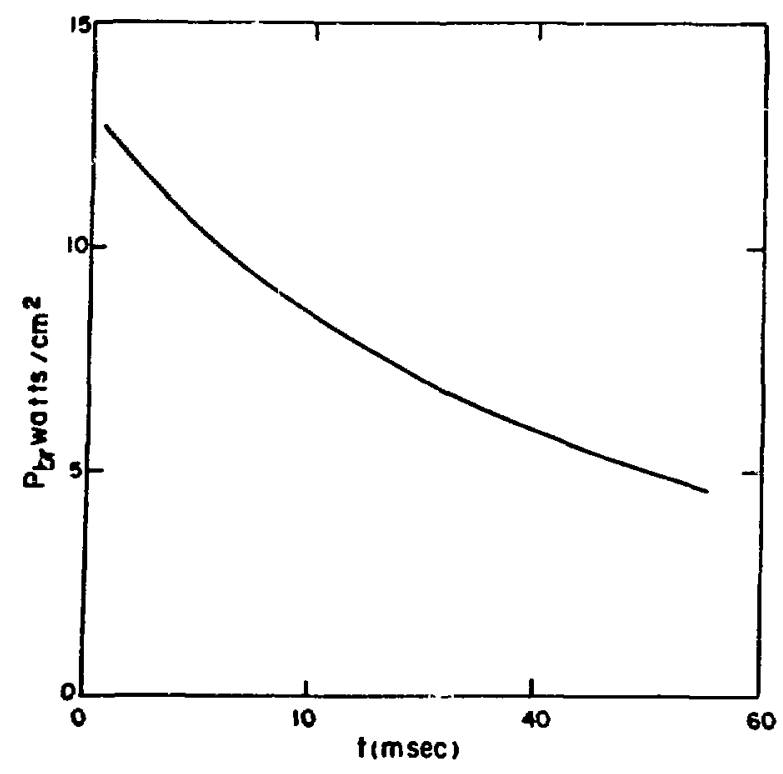

FIG. 4

Wall flux of bremsstrahlung radiation characteristic of a $5 \mathrm{keV}$ plasma for the parameters of Fig. 3 .

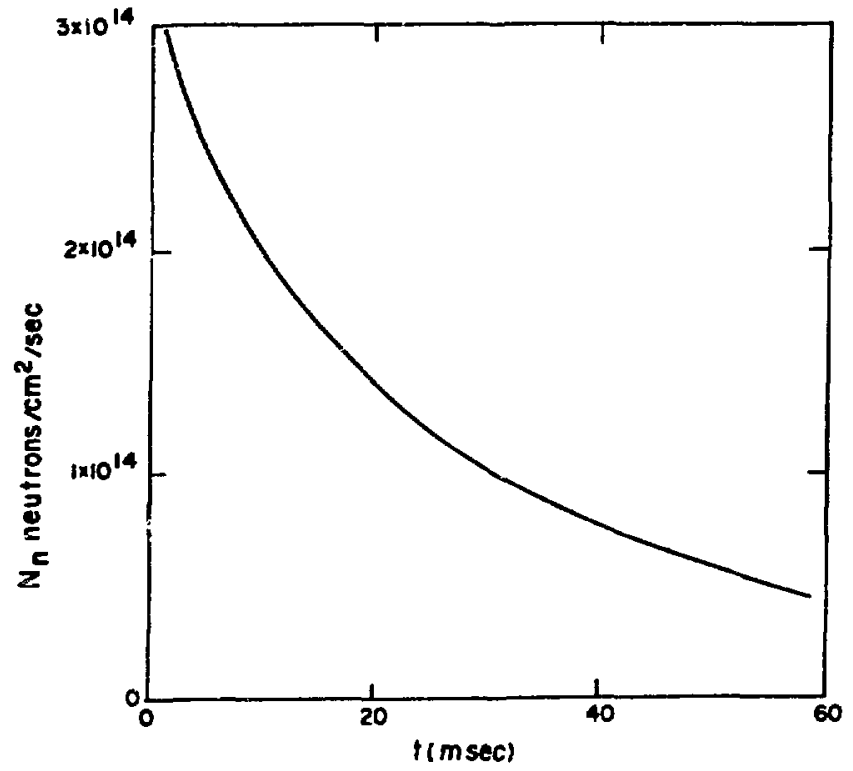

FIG 5

Neutron current to the wall for the parameters of Fig. 3 . 


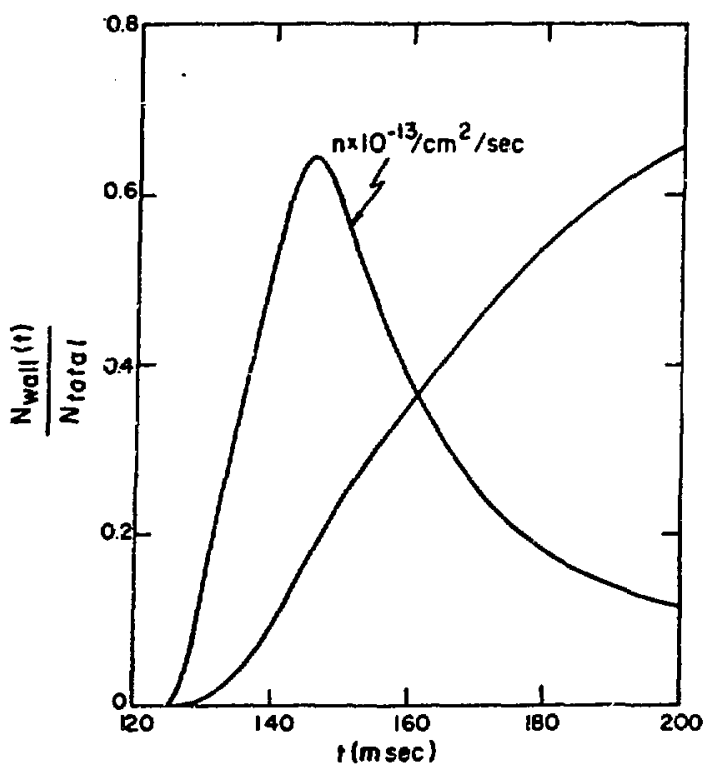

FIG. 6

Number of particles reaching the wall, $N$, normalized to the total number, and the derivative of this function giving the wall flux.

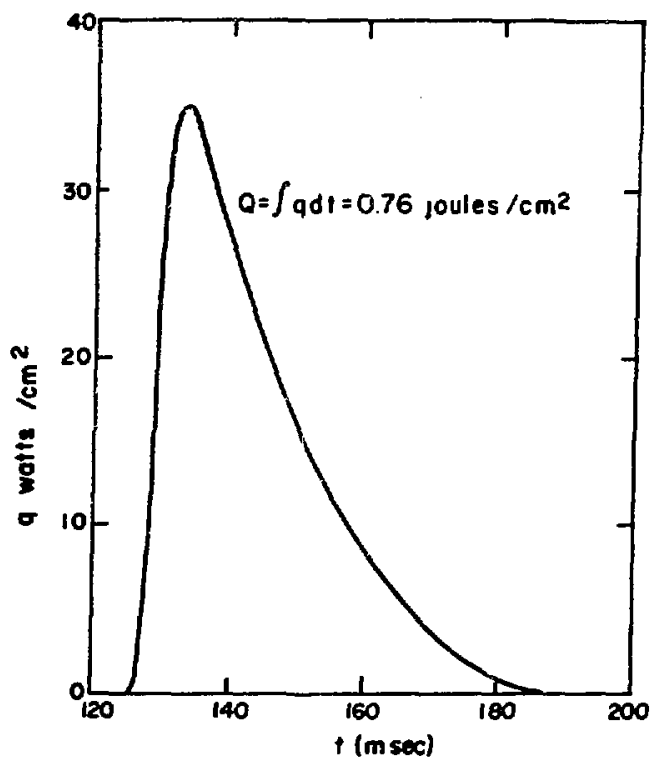

FIG. 7

Heat flux to the first wall and total heat per unit area for the parameters of Fig. 3 